\title{
Construction and screening of a glycosylphosphatidylinositol protein deletion library in Pichia pastoris
}

Pan Wang, Ying Lin, Chengjuan Zou, Fengguang Zhao, Shuli Liang, Suiping Zheng and Shuangyan Han*

\begin{abstract}
Background: Glycosylphosphatidylinositol (GPI)-anchored glycoproteins have diverse intrinsic functions in yeasts, and they also have different uses in vitro. In this study, the functions of potential GPI proteins in Pichia pastoris were explored by gene knockout approaches.

Results: Through an extensive knockout of GPI proteins in P. pastoris, a single-gene deletion library was constructed for 45 predicted GPI proteins. The knockout of proteins may lead to the activation of a cellular response named the 'compensatory mechanism', which is characterized by changes in the content and relationship between cell wall polysaccharides and surface proteins. Among the 45 deletion strains, five showed obvious methanol tolerance, four owned high content of cell wall polysaccharides, and four had a high surface hydrophobicity. Some advantages of these strains as production hosts were revealed. Furthermore, the deletion strains with high surface hydrophobicity were used as hosts to display Candida antarctica lipase B (CALB). The strain gcw22 $\Delta / C A L B-G C W 61$ showed excellent fermentation characteristics, including a faster growth rate and higher hydrolytic activity.
\end{abstract}

Conclusions: This GPI deletion library has some potential applications for production strains and offers a valuable resource for studying the precise functions of GPI proteins, especially their putative functions.

Keywords: GPI protein, Deletion, Phenotypic screen, Pichia pastoris

\section{Background}

Glycosylphosphatidylinositol (GPI)-anchored proteins are found in all eukaryotic cells. They harbor GPIanchoring machinery and utilize the anchor to express proteins on the cell surface. Precursors of GPI anchored proteins contain an N-terminal signal sequence for import into the ER and a C-terminal signal for GPI anchoring [1]. In yeast, the GPI anchor is essential for viability and maintenance of normal cell morphology $[2,3]$. These GPI-cell wall proteins (CWPs) can be grouped into different classes based on their functions. Some GPI proteins play a structural role and may provide stretch

\section{* Correspondence: syhan@scut.edu.cn}

Guangdong Key Laboratory of Fermentation and Enzyme Engineering, School of Biology and Biological Engineering, South China University of Technology, Guangzhou 510006, Guangdong, China resistance by interacting with glucans and other wall components or by interacting with each other through noncovalent bonds and disulfide bridges. Other GPI proteins may act as enzymes that make and break glycosidic linkages, and the rest are required for elaboration of the cell wall and its reshaping during bud emergence, cell separation, mating or entry into stationary phase.

Yeast cells elicit a rescue mechanism called the 'compensatory salvage response', which provides compensatory synthesis of cell wall material and changes the cross-linking type between cell wall polymers necessary for maintenance of cellular integrity and yeast survival $[4,5]$. Many responses occur under the control of the cell wall integrity signal transduction pathway and high osmotic glycerol pathway, which transmit wall stress signals from the cell surface, activate the Slt2 MAP kinase,

(c) The Author(s). 2020 Open Access This article is licensed under a Creative Commons Attribution 4.0 International License, which permits use, sharing, adaptation, distribution and reproduction in any medium or format, as long as you give appropriate credit to the original author(s) and the source, provide a link to the Creative Commons licence, and indicate if changes were made. The images or other third party material in this article are included in the article's Creative Commons licence, unless indicated otherwise in a credit line to the material. If material is not included in the article's Creative Commons licence and your intended use is not permitted by statutory regulation or exceeds the permitted use, you will need to obtain permission directly from the copyright holder. To view a copy of this licence, visit http://creativecommons.org/licenses/by/4.0/. The Creative Commons Public Domain Dedication waiver (http://creativecommons.org/publicdomain/zero/1.0/) applies to the data made available in this article, unless otherwise stated in a credit line to the data. 
and regulate the production and polarized delivery of various components to the site of cell wall remodeling. In particular, the cell wall structure needs to be remodeled under those stress conditions with a direct impact on cell wall integrity [6]. GPI proteins are also essential for maintaining cellular integrity. Quite a few GPI protein deletion strains have been constructed to explore cell properties and protein functions. For example, the loss of Ecm33p affects cell morphology, causes glycosylation defects, results in a short fermentation duration in either a synthetic medium or grape juice, and triggers the activation of the CWI MAPK pathway in S. cerevisiae $[7,8]$. A strain with knockouts of five GPI proteins (yps1 $\Delta$ yps $2 \Delta$ yps3 $\Delta$ yps6 $\Delta$ yps7 $\Delta$ ) did not show detectable growth defects under normal growth conditions but appeared to have an efficient ability to prevent the proteolytic degradation of hPTH in fed-batch cultivations compared with other combinations of deletion strains [9]. The stress-induced structural GPI protein SED1 was successfully knocked out in S. cerevisiae and used as a host strain. The strain produced an increasing amount of heterologous-displayed enzymes on the yeast cell surface using the SED1 anchoring system [10].

In view of the above phenotypic changes owing to the GPI protein deletion strains, potential strains playing positive roles in the expression of heterologous proteins, changing polysaccharide structure or content, and altering growth mode for survival should be discovered. By a comprehensive genome-wide search, 50 putative GPIanchored proteins in the $P$. pastoris genome were exploited in previous research, and 16 predicted GPI proteins could act as anchors to successfully display heterologous proteins on the cell surface [11]. This work opened the door to deeply study the role of GPI specifically in $P$. pastoris. $P$. pastoris is a widely used expression system with a strong promoter alcohol oxidase 1 (AOX1). With this system, recombinant protein expression can be induced 1000-fold upon methanol addition $[12,13]$. The display of enzymes on the surface of $P$. pastoris is an active topic in the field of whole-cell biocatalysts. However, the cell surface enzyme activity and protein amount are limited by some factors. For example, the anchorage position of the target protein in the cell wall is an important factor that maximizes the capabilities of engineered yeast cells [14]. Therefore, the functions of GPI-anchored proteins on cell surface display should be further explored.

However, the physiological roles of more than $70 \%$ of these predicted GPI-anchored proteins in P. pastoris are unknown. In the present study, we hope to obtain more information about the GPI proteins in $P$. pastoris. The function of GPI proteins in $P$. pastoris could also provide more information on other excellent expression systems $[15,16]$. According to the compensatory salvage response, the deletion strains could elicit a series of phenotypic changes, including changes in the cell wall polysaccharide content and protein types. Therefore, a GPI protein deletion library was constructed by knocking out a single predicted GPI protein-encoding gene in $P$. pastoris. Finally, 45 GPI protein deletion strains were successfully obtained, and their characteristics were investigated.

\section{Results \\ Construction of a GPI protein deletion library with the Cre/loxp system}

According to the amino acid sequence characteristics of GPI-anchored proteins, Zhang et al. predicted and screened 50 potential GPI-anchored proteins in P. pastoris [11]. To explore the functions of these proteins, a GPI protein deletion library with each predicted protein knocked out with the Cre/loxP system was contructed. To maximize the efficiency of gene replacement, the gene knockout expression cassette contained $\sim 700 \mathrm{bp}$ homologous flanking regions for every gene. Finally, 17 gene knockout expression cassettes were successfully transformed into GS115. To significantly increase homologous recombination efficiencies in P. pastoris, the endogenous gene ku70 homolog, a key player in nonhomologous-end-joining (NHEJ) repair, was deleted, and the strain GS115 ku70 was constructed [17]. Another 28 gene knockout expression cassettes were transformed into the strain GS115 ku70, and the recombination efficiency significantly improved. Thus, 45 GPI-deficient strains were successfully obtained. The detailed information is shown in Table 1.

\section{Growth of deletion library strains with different carbon sources}

Yeast can grow on different carbon sources, which are known to influence their growth behavior. To explore the growth characteristics of the deletion strains, four different carbon source cultures, YPD, YPG, YPM and BMMY, were used. The specific growth rates of all the strains are listed in Table 2. These GPI deletion strains grew normally on YPD and YPG media compared with the control strain, indicating that the knocked out genes are not necessary for yeast cell growth. The utilization rates of different carbon sources showed wide variations. Generally, the cells grew better on the carbon sources with glucose or glycerol but grew poorly with methanol. As a methylotrophic yeast, $P$. pastoris has been given great attention for its ability to utilize low-cost methanol as a sole carbon source to express high levels of recombinant proteins. However, high methanol concentrations often result in severe growth defects, which presents serious issues for industrial applications. Five strains in the GPI deletion library, gcw13 $\Delta$, gcw17 $\Delta$, gcw19 $\Delta$, gcw21 $\Delta$, 
Table 1 The gene information of GPI proteins in deletion library strains

\begin{tabular}{|c|c|c|c|c|}
\hline \multirow[t]{2}{*}{ Strains } & \multicolumn{4}{|c|}{ Deletion gene } \\
\hline & Gene name & Size (AA) & NCBI BLAST & Domain description \\
\hline $\operatorname{gcw} 1 \Delta$ & GCW1 & 538 & $\beta$-1,3-Glucanosyltransferase & Glucanosyltransferase; X8; Glycoside hydrolase \\
\hline $\operatorname{gcw} 2 \Delta$ & GCW2 & 535 & $\beta$-1,3-Glucanosyltransferase & Glucanosyltransferase; X8; Glycoside hydrolase \\
\hline ku70 $\Delta$ gcw3 $\Delta$ & GCW3 & 307 & Hypothetical protein & Superoxide dismutase, Cu/Zn binding domain \\
\hline gcw $4 \Delta$ & GCW4 & 266 & Hypothetical protein & No hits found \\
\hline $\operatorname{gcw} 5 \Delta$ & GCW5 & 203 & Hypothetical protein & Flocculin type 3 repeat \\
\hline gcw6 $\Delta$ & GCW6 & 448 & Cell wall protein & Glycoside hydrolase, predicted $\mathrm{CRH}$; Concanavalin A-like lectin/glucanases \\
\hline gcw7 $\Delta$ & GCW7 & 593 & Aspartic protease & Peptidase A1; Aspartic peptidase \\
\hline ku70 $\Delta$ gcw8 $\Delta$ & GCW8 & 448 & Putative mannosidase & $\begin{array}{l}\text { Glycoside hydrolase; Six-hairpin glycosidase; Mannanendo-1,6-alpha- } \\
\text { mannosidase }\end{array}$ \\
\hline gcw10 $\Delta$ & GCW10 & 251 & Hypothetical protein & No hits found \\
\hline gcw $12 \Delta$ & GCW12 & 233 & CFEM protein & Extracellular membrane protein, CFEM domain \\
\hline $\operatorname{gcw} 13 \Delta$ & GCW13 & 294 & Hypothetical protein & No hits found \\
\hline gcw14 $\Delta$ & GCW14 & 135 & Hypothetical protein & No hits found \\
\hline gcw $15 \Delta$ & GCW15 & 237 & Hypothetical protein & No hits found \\
\hline gcw16 $\Delta$ & GCW16 & 1416 & Mucin-like protein & Cellulose-binding domain; Uncharacterized domain Flo11-related \\
\hline gcw17 & GCW17 & 259 & Hypothetical protein & No hits found \\
\hline- & GCW18 & 1667 & Hypothetical protein & Peptidase S8/S53 domain; PT repeat, subtilisin-related \\
\hline gcw19 & GCW19 & 148 & Hypothetical protein & No hits found \\
\hline $\operatorname{gcw} 21 \Delta$ & GCW21 & 233 & Hypothetical protein & No hits found \\
\hline gcw $22 \Delta$ & GCW22 & 839 & Hypothetical protein & Uncharacterized domain Flo11-related, N-terminal; Flocculin type 3 repeat \\
\hline- & GCW23 & 418 & Cell wall protein & Receptor L domain-like \\
\hline ku70 $\Delta$ gcw24 $\Delta$ & GCW24 & 261 & Hypothetical protein & No hits found \\
\hline ku70 $\Delta$ gcw25 $\Delta$ & GCW25 & 595 & Hypothetical protein & Flocculin type 3 repeat \\
\hline ku70 $\Delta$ gcw26 $\Delta$ & GCW26 & 724 & Hypothetical protein & GLEYA adhesin domain \\
\hline ku70 $\Delta$ gcw28 $\Delta$ & GCW28 & 587 & Mucin-like protein & Adhesion domain, bacterial \\
\hline- & GCW29 & 1474 & Hypothetical protein & PT repeat; Adhesion domain, bacterial \\
\hline ku70 $\Delta$ gcw30 $\Delta$ & GCW30 & 229 & Hypothetical protein & No hits found \\
\hline ku70 $\Delta$ gcw31 $\Delta$ & GCW31 & 562 & Hypothetical protein & Peptidase A1; Aspartic peptidase \\
\hline ku70 $\Delta$ gcw32 $\Delta$ & GCW32 & 216 & Hypothetical protein & No hits found \\
\hline- & GCW33 & 527 & Aspartic protease & Peptidase A1; Aspartic peptidase \\
\hline ku70 $\Delta$ gcw34 $\Delta$ & GCW34 & 473 & Aspartic protease & Peptidase A1; Aspartic peptidase \\
\hline ku70 $\Delta$ gcw35 $\Delta$ & GCW35 & 582 & Putative aspartic protease & Peptidase A1; Aspartic peptidase \\
\hline ku70 $\Delta$ gcw36 $\Delta$ & GCW36 & 468 & Hypothetical protein & PT repeat \\
\hline ku70 $\Delta$ gcw37 $\Delta$ & GCW37 & 234 & Hypothetical protein & No hits found \\
\hline ku70 $\Delta$ gcw39 $\Delta$ & GCW39 & 612 & Aspartic protease & Peptidase A1; Aspartic peptidase \\
\hline ku70 $\Delta$ gcw $42 \Delta$ & GCW42 & 248 & Hypothetical protein & No hits found \\
\hline ku70 $\Delta$ gcw43 $\Delta$ & GCW43 & 443 & Hypothetical protein & EGF receptor, L domain \\
\hline ku70 $\Delta$ gcw $45 \Delta$ & GCW45 & 409 & Hypothetical protein & PT repeat; Flocculin type 3 repeat \\
\hline ku70 $\Delta$ gcw $46 \Delta$ & GCW46 & 633 & Phospholipase B & Lysophospholipase, catalytic domain; Acyl transferase/acyl hydrolase \\
\hline ku70 $\Delta$ gcw $48 \Delta$ & GCW48 & 369 & Putative protease & SUN Family \\
\hline ku70 $\Delta$ gcw $49 \Delta$ & GCW49 & 327 & Hypothetical protein & No hits found \\
\hline- & GCW50 & 454 & Hypothetical protein & No hits found \\
\hline ku70 $\Delta$ gcw51 $\Delta$ & GCW51 & 211 & Hypothetical protein & No hits found \\
\hline
\end{tabular}


Table 1 The gene information of GPI proteins in deletion library strains (Continued)

\begin{tabular}{lllll}
\hline Strains & \multicolumn{3}{l}{ Deletion gene } & \\
\cline { 2 - 5 } Gene name & Size (AA) & NCBI BLAST & Domain description \\
\hline ku70 $\Delta$ gcw52 $\Delta$ & GCW52 & 365 & Hypothetical protein & No hits found \\
ku70 $\Delta$ gcw53 $\Delta$ & GCW53 & 354 & Hypothetical protein & No hits found \\
ku70 $\Delta$ gcw54 $\Delta$ & GCW54 & 611 & Hypothetical protein & No hits found \\
ku70 $\Delta$ gcw56 $\Delta$ & GCW56 & 400 & Hypothetical protein & No hits found \\
ku70 $\Delta$ gcw58 $\Delta$ & GCW58 & 194 & Hypothetical protein & Stress-induced protein SRP1/TIP1 \\
ku70 $\Delta$ gcw59 $\Delta$ & GCW59 & 469 & Putative chitin transglycosidase & Glycoside hydrolase, predicted CRH1;Concanavalin A-like lectin/glucanases \\
ku70 $\Delta$ gcw60 $\Delta$ & GCW60 & 599 & Aspartic protease & Peptidase A1; Aspartic peptidase \\
gcw61 $\Delta$ & GCW61 & 65 & Hypothetical protein & No hits found \\
\hline
\end{tabular}

and gcw $22 \Delta$, showed obvious variations in the media supplemented with methanol as the carbon source, including the media YPM, 1\% BMMY, 2\% BMMY and 3\% BMMY. Significantly accelerated specific growth rates were observed for the above five strains. Furthermore, the cell growth of these five strains in BMMY medium with shake flasks also exhibited a remarkable growth advantage (Fig. 1), which was similar to the growth state in 96-well plates.

\section{Remodeling of cell wall polysaccharides in the deletion library}

Cell wall polysaccharides mainly contain polymers of mannose, glucose and $\mathrm{N}$-acetylglucosamine, but the synthesis of cell wall polysaccharides is also dynamic and shifts to facilitate the loosening and strengthening of the cell wall. A lack of GPI proteins may lead to the remodeling of yeast cell wall polysaccharides. The content of cell wall polysaccharides in the deletion library was

Table $\mathbf{2}$ The specific growth rate $\mu$ of deletion library strains

\begin{tabular}{|c|c|c|c|c|c|c|c|c|c|c|c|c|c|}
\hline Strains & YPD & YPG & YPM & $1 \% \mathrm{BMMY}$ & $2 \% \mathrm{BMMY}$ & $3 \% \mathrm{BMMY}$ & Strains & YPD & YPG & YPM & 1\%BMMY & 2\%BMMY & 3\%BMMY \\
\hline GS115 & 0.288 & 0.201 & 0.119 & 0.129 & 0.094 & 0.060 & ku70 $\Delta$ gcw28 $\Delta$ & 0.244 & 0.165 & 0.099 & 0.084 & 0.063 & 0.054 \\
\hline gcw1 $\Delta$ & 0.285 & 0.197 & 0.110 & 0.144 & 0.116 & 0.076 & ku70 $\Delta$ gcw30 $\Delta$ & 0.271 & 0.191 & 0.095 & 0.103 & 0.118 & 0.054 \\
\hline $\operatorname{gcw} 2 \Delta$ & 0.338 & 0.243 & 0.130 & 0.171 & 0.140 & 0.080 & ku70 $\Delta$ gcw31 $\Delta$ & 0.281 & 0.212 & 0.096 & 0.127 & 0.103 & 0.053 \\
\hline gcw4 $\Delta$ & 0.271 & 0.220 & 0.107 & 0.156 & 0.101 & 0.055 & ku70 $\Delta$ gcw32 $\Delta$ & 0.294 & 0.216 & 0.083 & 0.117 & 0.099 & 0.052 \\
\hline $\operatorname{gcw} 5 \Delta$ & 0.308 & 0.215 & 0.098 & 0.121 & 0.108 & 0.067 & ku70 $\Delta$ gcw34 $\Delta$ & 0.301 & 0.236 & 0.080 & 0.131 & 0.118 & 0.036 \\
\hline gcw6 $\Delta$ & 0.287 & 0.208 & 0.116 & 0.146 & 0.099 & 0.057 & ku70 $\Delta$ gcw35 $\Delta$ & 0.287 & 0.202 & 0.086 & 0.110 & 0.103 & 0.045 \\
\hline $\operatorname{gcw} 7 \Delta$ & 0.287 & 0.193 & 0.104 & 0.106 & 0.114 & 0.060 & ku70 $\Delta$ gcw36 $\Delta$ & 0.268 & 0.182 & 0.092 & 0.092 & 0.069 & 0.060 \\
\hline gcw10 $\Delta$ & 0.254 & 0.152 & 0.120 & 0.139 & 0.109 & 0.054 & ku70 $\Delta$ gcw37 $\Delta$ & 0.315 & 0.227 & 0.102 & 0.129 & 0.114 & 0.070 \\
\hline gcw $12 \Delta$ & 0.280 & 0.212 & 0.115 & 0.188 & 0.154 & 0.066 & ku70 $\Delta$ gcw39 $\Delta$ & 0.269 & 0.211 & 0.097 & 0.093 & 0.095 & 0.051 \\
\hline $\operatorname{gcw} 13 \Delta$ & 0.316 & 0.214 & 0.165 & 0.212 & 0.207 & 0.185 & ku70 $\Delta$ gcw42 $\Delta$ & 0.303 & 0.229 & 0.105 & 0.091 & 0.071 & 0.048 \\
\hline gcw14 $\Delta$ & 0.276 & 0.167 & 0.101 & 0.126 & 0.100 & 0.065 & ku70 $\Delta$ gcw43 $\Delta$ & 0.278 & 0.166 & 0.097 & 0.097 & 0.081 & 0.049 \\
\hline gcw15 $\Delta$ & 0.339 & 0.204 & 0.124 & 0.148 & 0.113 & 0.050 & ku70 $\Delta$ gcw $45 \Delta$ & 0.280 & 0.230 & 0.100 & 0.133 & 0.109 & 0.053 \\
\hline gcw16 $\Delta$ & 0.282 & 0.222 & 0.130 & 0.103 & 0.186 & 0.060 & ku70 $\Delta$ gcw $46 \Delta$ & 0.265 & 0.182 & 0.105 & 0.095 & 0.094 & 0.058 \\
\hline gcw17 $\Delta$ & 0.283 & 0.220 & 0.166 & 0.215 & 0.189 & 0.168 & ku70 $\Delta$ gcw48 $\Delta$ & 0.282 & 0.206 & 0.089 & 0.121 & 0.106 & 0.045 \\
\hline gcw19 $\triangle$ & 0.302 & 0.180 & 0.181 & 0.237 & 0.265 & 0.186 & ku70 $\Delta$ gcw49 $\Delta$ & 0.273 & 0.215 & 0.096 & 0.094 & 0.111 & 0.050 \\
\hline $\operatorname{gcw} 21 \Delta$ & 0.287 & 0.194 & 0.167 & 0.214 & 0.228 & 0.160 & ku70 $\Delta$ gcw51 $\Delta$ & 0.296 & 0.219 & 0.093 & 0.114 & 0.124 & 0.048 \\
\hline gcw22 $\Delta$ & 0.308 & 0.223 & 0.155 & 0.222 & 0.192 & 0.176 & ku70 $\Delta$ gcw52 $\Delta$ & 0.245 & 0.188 & 0.139 & 0.094 & 0.064 & 0.049 \\
\hline gcw61 $\Delta$ & 0.309 & 0.216 & 0.115 & 0.130 & 0.123 & 0.082 & ku70 $\Delta$ gcw53 $\Delta$ & 0.273 & 0.197 & 0.105 & 0.126 & 0.081 & 0.063 \\
\hline GS115 ku70 $\triangle$ & 0.258 & 0.197 & 0.093 & 0.118 & 0.090 & 0.050 & ku70 $\Delta$ gcw54 $\Delta$ & 0.252 & 0.203 & 0.127 & 0.084 & 0.076 & 0.043 \\
\hline ku70 $\Delta$ gcw3 $\Delta$ & 0.226 & 0.212 & 0.088 & 0.075 & 0.065 & 0.066 & ku70 $\Delta$ gcw $56 \Delta$ & 0.250 & 0.189 & 0.110 & 0.085 & 0.073 & 0.057 \\
\hline ku70 $\Delta$ gcw8 $\Delta$ & 0.255 & 0.221 & 0.061 & 0.090 & 0.103 & 0.046 & ku70 $\Delta$ gcw58 $\Delta$ & 0.295 & 0.240 & 0.120 & 0.085 & 0.091 & 0.070 \\
\hline ku70 $\Delta$ gcw24 $\Delta$ & 0.270 & 0.202 & 0.091 & 0.121 & 0.112 & 0.050 & ku70 $\Delta$ gcw59 $\Delta$ & 0.251 & 0.181 & 0.127 & 0.076 & 0.059 & 0.035 \\
\hline ku70 $\Delta$ gcw25 $\Delta$ & 0.265 & 0.212 & 0.092 & 0.087 & 0.118 & 0.047 & ku70 $\Delta$ gcw60 $\Delta$ & 0.275 & 0.208 & 0.118 & 0.116 & 0.104 & 0.067 \\
\hline ku70 $\Delta$ gcw26 $\Delta$ & 0.264 & 0.185 & 0.101 & 0.119 & 0.098 & 0.047 & & & & & & & \\
\hline
\end{tabular}



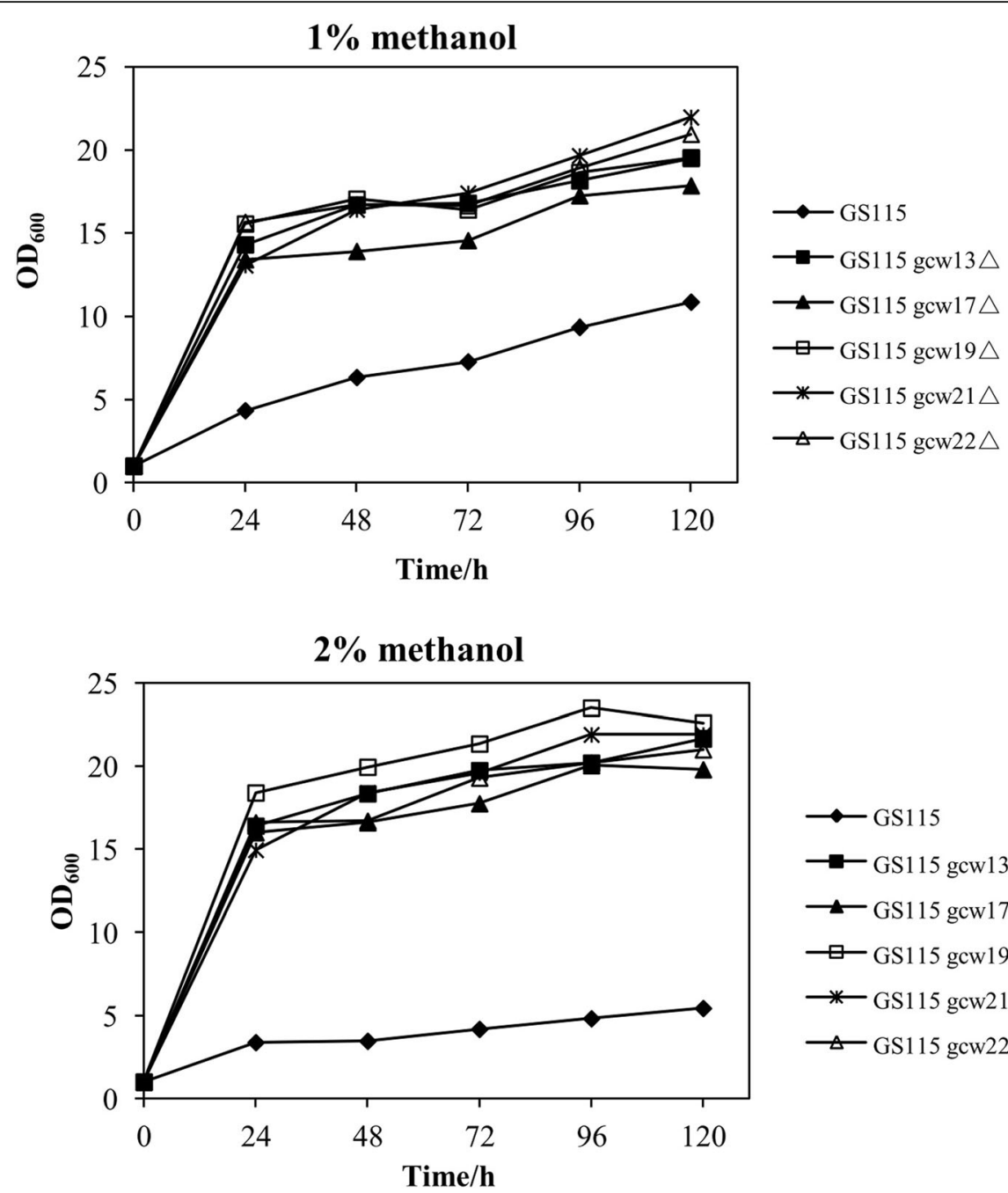

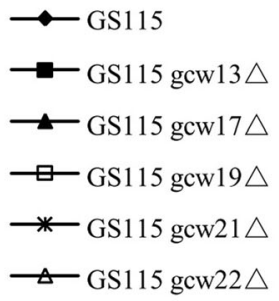

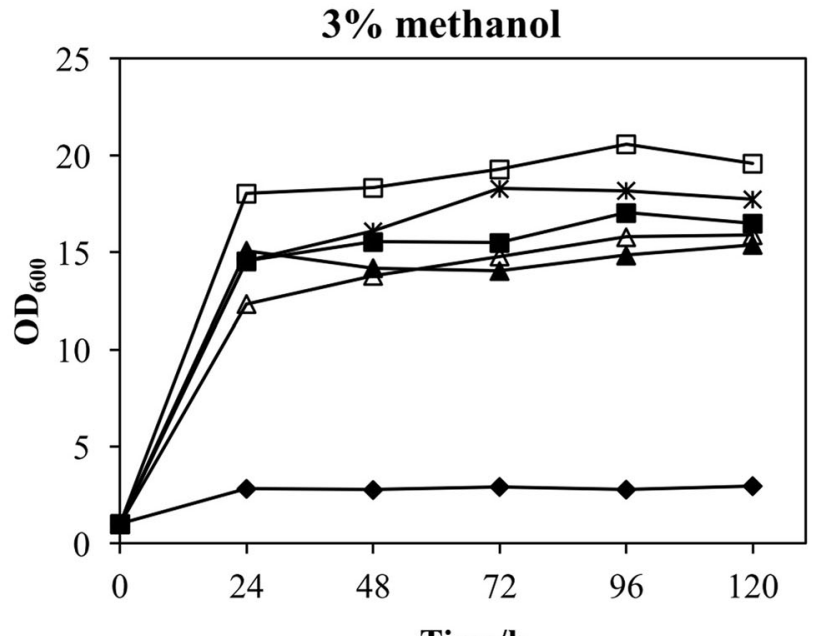

$\longrightarrow$ GS115

$\rightarrow-G S 115$ gcwl3 $\triangle$

$\longrightarrow$ GS115 gcw17 $\triangle$

七-GS115 gcw19 $\triangle$

* GS115 gew21 $\triangle$

$\triangle$ GS115 gcw22 $\triangle$

Time/h

Fig. 1 Fermentation curve of deletion strains in media with different methanol concentrationsCells were cultured in BMMY medium for $120 \mathrm{~h}$, and methanol was added every $24 \mathrm{~h}$ 
measured, and changes in cell wall chitin, dextran or mannan were observed in Fig. 2.

For strain ku70 $\Delta$ gcw51 $\Delta$, the contents of glucan and mannan increased by 27 and 33\%, respectively. For strain ku70 $\Delta$ gcw54 $\Delta$, the contents of glucan and mannan increased by 26 and 39\%, respectively. In addition, the glucan content of strain ku70 $\Delta$ gcw $8 \Delta$ increased by $40 \%$, and the mannan content of strain gcw $1 \Delta$ increased by $34 \%$. These results suggest that the deletion of these GPI proteins leads to remodeling of the yeast cell wall polysaccharide.

\section{Screening surface hydrophobic strains in deletion library strains as hosts to obtain recombinant strains with high hydrolytic activity}

The surface hydrophobicity of the strains in the deletion library is listed in Table 3 . Among all the deletion strains, the strains gcw $13 \Delta$, gcw $22 \Delta$, ku70 $\Delta$ gcw30 $\Delta$, and ku70 $\Delta$ gcw53 $\Delta$ showed higher surface hydrophobic properties. Moreover, the protein GRAVY value was calculated by the sum of hydropathy values of all amino acids divided by the protein length. After identifying the strains with higher surface hydrophobic properties, some deleted GPI proteins, such as GCW13, GCW22, GCW30 and GCW53, were found to correspond to hydrophobic strains that seemed to have stronger hydrophilic properties by the GRAVY calculator.

To explore the application potential of these screened hydrophobic yeast cells, the recombinant plasmid pZCALB-GCW61 was constructed. Then, the plasmid was transformed into strains GS115 and GS115 ku70 $\Delta$ as control strains. The fermentation curves are shown in Fig. 3.
The growth of strain gcw22 $\Delta /$ CALB-GCW61 was faster than that of other strains in the first $72 \mathrm{~h}$ and then soon reached a plateau. The specific growth rate, $\mu$, of strain gcw22 $\Delta /$ CALB-GCW61 was $0.33 \mathrm{~h}^{-1}$, corresponding to an increase of $23 \%$ compared with strain GS115/CALBGCW61 $\left(0.27 \mathrm{~h}^{-1}\right)$. Strain gcw13 $/$ CALB-GCW61 maintained a similar growth rate as the control strain. The growth rate of the other three strains, ku70 $\Delta$ gcw30 $\Delta /$ CALB-GCW61 and ku70 $\Delta$ gcw53 $\Delta$ /CALB-GCW61, slowed down significantly after $48 \mathrm{~h}$. The lipase hydrolysis activity of all the recombinant strains increased except strain gcw13 $\Delta / C A L B-G C W 61$. In particular, the hydrolysis activity of strain gcw $22 \Delta /$ CALB-GCW61 increased by $69 \%$ after fermentation for $120 \mathrm{~h}$. The surface hydrophobicity of the recombinant strain was also detected. This result was consistent with the enzyme activity results, and strain gcw $22 \Delta /$ CALB-GCW61 showed the highest surface hydrophobicity. Although strain ku70 $\Delta$ gcw30 $\Delta / C A L B-G C W 61$ had the same surface hydrophobicity, the lipase activity did not significantly change (Table 3 ).

\section{Discussion}

Among the 50 putative GPI proteins in P. pastoris, more than $70 \%$ of them have unknown physiologic functions. Using the Cre/loxP system, a library of 45 deletion strains representing almost $90 \%$ of the candidate GPI genes was constructed. This is the largest library of GPI protein deletions so far, and this library is a significant step towards unveiling the roles of all the GPI proteins of $P$. pastoris. In this study, the deletion library was subjected to a series of phenotypic tests, and some strains with potential applications were found and evaluated.

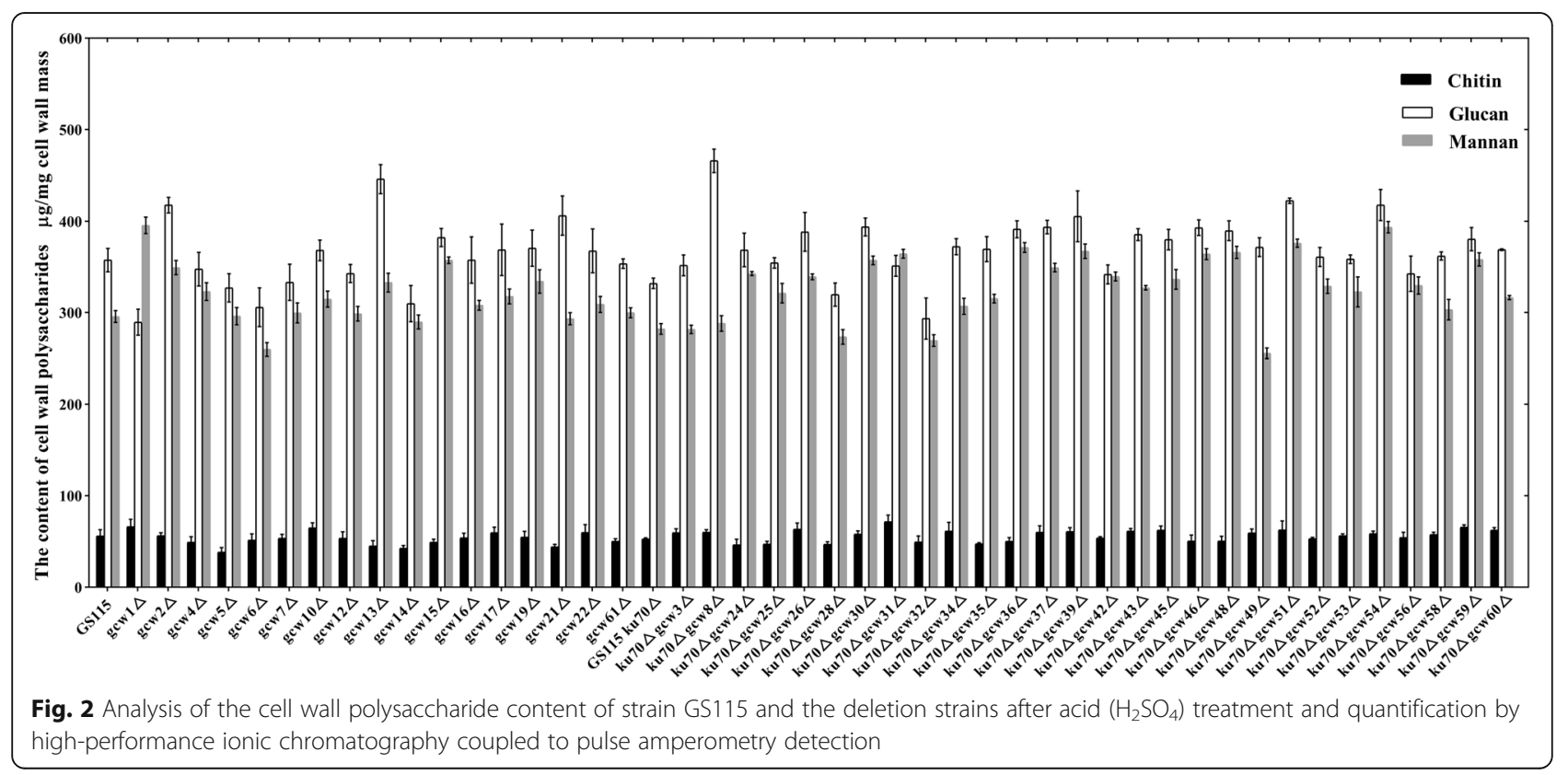


Table 3 The surface hydrophobicity of deletion library strains and the GRAVY values of deleted GPI proteins

\begin{tabular}{|c|c|c|c|c|c|}
\hline Strains & hydrophobicity & GRAVY* & Strains & hydrophobicity & GRAVY \\
\hline GS115 & $0.24 \pm 0.07$ & - & ku70 $\Delta$ gcw $28 \Delta$ & $0.44 \pm 0.10$ & 0.02 \\
\hline $\operatorname{gcw} 1 \Delta$ & $0.18 \pm 0.11$ & -0.14 & ku70 $\Delta$ gcw $30 \Delta$ & $0.49 \pm 0.03$ & -0.73 \\
\hline $\operatorname{gcw} 2 \Delta$ & $0.38+0.07$ & -0.21 & ku70 $\Delta$ gcw31 $\Delta$ & $0.20+0.02$ & -0.11 \\
\hline gcw $4 \Delta$ & $0.43 \pm 0.04$ & 0.21 & ku70 $\Delta$ gcw32 $\Delta$ & $0.49 \pm 0.10$ & 0.23 \\
\hline $\operatorname{gcw} 5 \Delta$ & $0.34 \pm 0.07$ & 0.06 & ku70 $\Delta$ gcw34 $\Delta$ & $0.28 \pm 0.07$ & -0.22 \\
\hline gcw6 $\Delta$ & $0.34 \pm 0.02$ & -0.42 & ku70 $\Delta$ gcw $35 \Delta$ & $0.13+0.01$ & -0.05 \\
\hline gcw7 $\Delta$ & $0.33 \pm 0.09$ & -0.17 & ku70 $\Delta$ gcw36 $\Delta$ & $0.37 \pm 0.03$ & -0.56 \\
\hline gcw10 $\Delta$ & $0.44 \pm 0.00$ & -0.28 & ku70 $\Delta$ gcw37 $\Delta$ & $0.32 \pm 0.05$ & 0.20 \\
\hline gcw12 $\Delta$ & $0.25+0.04$ & 0.16 & ku70 $\Delta$ gcw39 $\Delta$ & $0.42 \pm 0.05$ & -0.12 \\
\hline gcw13 $\Delta$ & $0.47 \pm 0.00$ & -0.50 & ku70 $\Delta$ gcw $42 \Delta$ & $0.27+0.06$ & 0.13 \\
\hline gcw14 $\Delta$ & $0.43+0.09$ & 0.20 & ku70 $\Delta$ gcw $43 \Delta$ & $0.49 \pm 0.01$ & -0.08 \\
\hline gcw $15 \Delta$ & $0.45 \pm 0.03$ & -0.32 & ku70 $\Delta$ gcw $45 \Delta$ & $0.36 \pm 0.08$ & -0.64 \\
\hline gcw16 $\Delta$ & $0.17+0.05$ & -0.62 & ku70 $\Delta$ gcw $46 \Delta$ & $0.18+0.01$ & -0.22 \\
\hline gcw17 $\Delta$ & $0.14+0.02$ & -0.17 & ku70 $\Delta$ gcw $48 \Delta$ & $0.27+0.02$ & -0.26 \\
\hline gcw19 & $0.20+0.00$ & 0.13 & ku70 $\Delta$ gcw $49 \Delta$ & $0.31 \pm 0.04$ & -0.34 \\
\hline $\operatorname{gcw} 21 \Delta$ & $0.27+0.05$ & 0.03 & ku70 $\Delta$ gcw51 $\Delta$ & $0.30 \pm 0.02$ & -0.20 \\
\hline gcw22 $\Delta$ & $0.57 \pm 0.09$ & -0.68 & ku $70 \Delta$ gcw52 $\Delta$ & $0.18 \pm 0.06$ & -0.49 \\
\hline gcw61 $\Delta$ & $0.45 \pm 0.02$ & 0.55 & ku70 $\Delta$ gcw $53 \Delta$ & $0.48 \pm 0.09$ & -0.37 \\
\hline GS115 ku70 & $0.16 \pm 0.03$ & - & ku70 $\Delta$ gcw $54 \Delta$ & $0.25+0.02$ & -0.21 \\
\hline ku $70 \Delta$ gcw3 $\Delta$ & $0.28 \pm 0.11$ & -0.54 & ku70 $\Delta$ gcw56 $\Delta$ & $0.32 \pm 0.07$ & 0.05 \\
\hline ku70 $\Delta$ gcw8 $\Delta$ & $0.29+0.05$ & -0.04 & ku70 $\Delta$ gcw $58 \Delta$ & $0.35+0.03$ & -0.27 \\
\hline ku70 $\Delta$ gcw24 $\Delta$ & $0.21 \pm 0.04$ & -0.13 & ku70 $\Delta$ gcw59 $\Delta$ & $0.26+0.03$ & -0.36 \\
\hline ku $70 \Delta$ gcw $25 \Delta$ & $0.32 \pm 0.07$ & 0.01 & ku70 $\Delta$ gcw60 $\Delta$ & $0.42+0.01$ & -0.08 \\
\hline ku70 $\Delta$ gcw26 $\Delta$ & $0.22 \pm 0.06$ & -0.18 & & & \\
\hline
\end{tabular}

GRAVY Grand average of hydropathicity

The methylotrophic yeast $P$. pastoris is currently a powerful system for the production of recombinant heterogeneous proteins [18]. The control of the methanol concentration in an appropriate range can promote yeast growth and protein expression. However, higher methanol concentrations often lead to the accumulation of formaldehyde, retard growth and decrease the observed biomass yield $[19,20]$. However, a lower methanol concentration means less carbon source, resulting in a decrease in protein production. Therefore, improving the tolerance of the strain to methanol and increasing the rate of methanol utilization for $P$. pastoris have been in great demand. The strategy can also shorten the period of yeast culture, increase the production intensity and reduce the production cost. Five strains in this deletion library, gcw13 $\Delta$, gcw17 $\Delta$, gcw19 $\Delta$, gcw21 $\Delta$, and gcw22 $\Delta$, showed obvious methanol tolerance characteristics in YPM and BMMY media supplemented with different methanol concentrations. It could be speculated that these deletion strains triggered remodeling of cell wall protein composition and led to an increase in cell tolerance to methanol because the cell membrane and cell wall were major targets of methanol toxicity [21]. Studies have demonstrated that GCW13 suppresses the expression of GAP1, which encodes a general amino acid permease, and facilitates the endocytosis of GAP1 in methanol. The deletion of the GCW13 gene derepresses GAP1-dependent uptake of amino acids and shows better methanol tolerance [22]. In C. glutamicum, increased methanol tolerance may be relevant to two point mutations: one leading to the amino acid exchange A165T of the O-acetylhomoserine sulfhydrolase MetY and the other leading to shortening of the CoA transferase Cat (Q342*) [23]. In S. cerevisiae, a large number of genes required for methanol tolerance are attributed to vacuolar function, transcription and some unknown function [24]. In addition to the protein GCW13, the physiologic functions of other GPI proteins, GCW17, GCW19, GCW21 and GCW22, need to be explored further.

Mannoproteins and $\beta$-glucans represent the majority of the polysaccharides in the yeast cell wall. In recent years, yeast polysaccharides have been proven to be immunostimulant molecules for human and animal health due to their ability to enhance and stimulate the 


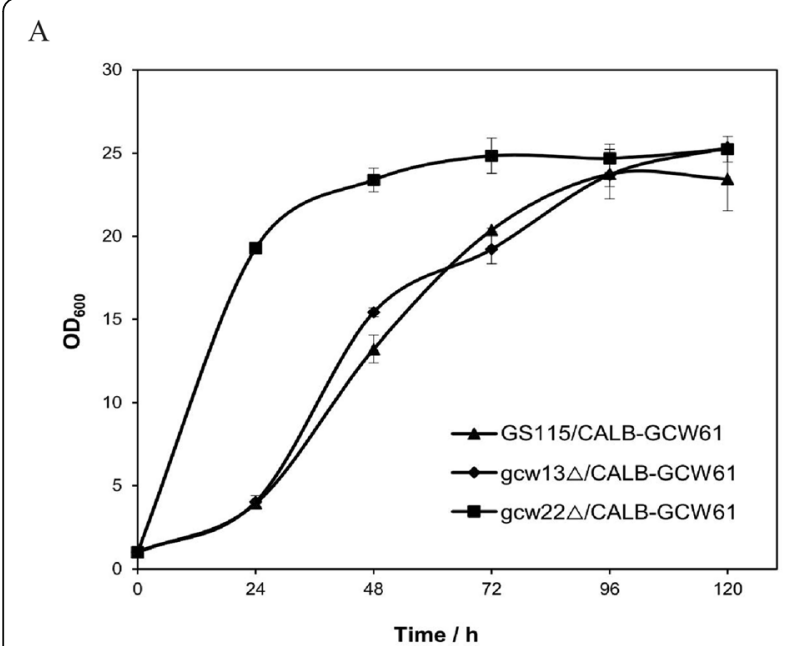

B

C
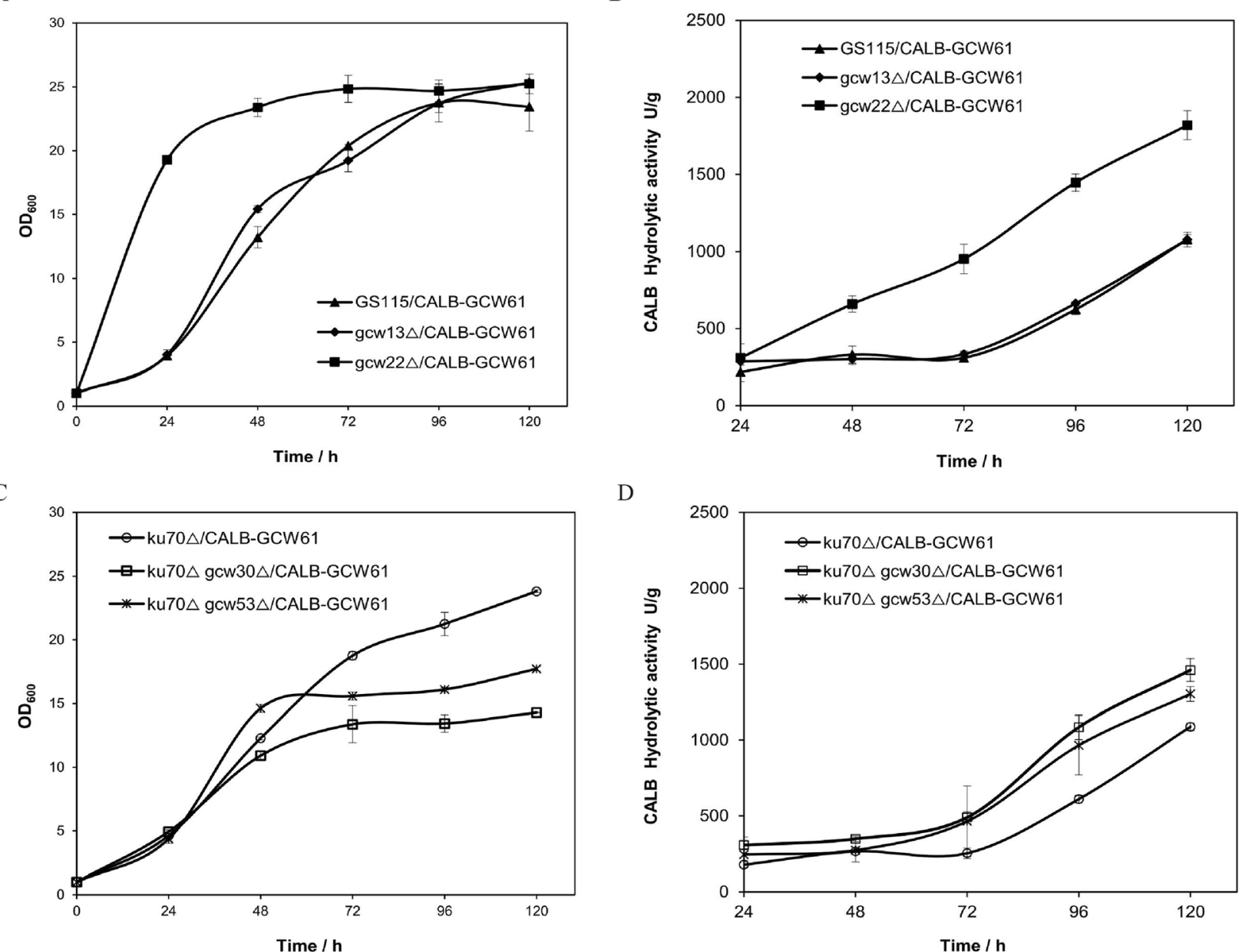

D

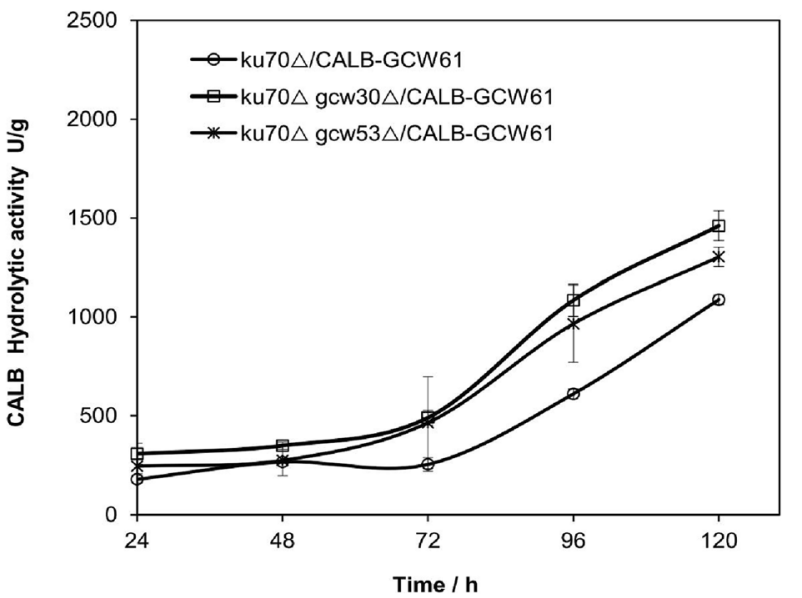

Fig. 3 Growth characteristics and hydrolytic activity of recombinant strains. Recombinant Cells were induced to express CALB in BMMY medium containing $1 \%(\mathrm{~V} / \mathrm{V})$ methanol for $120 \mathrm{~h}$. The hydrolytic activity was measured using pNPB as the substrate. $\mathbf{a}$ and $\mathbf{c}$, Growth curves of deletion strains. $\mathbf{b}$ and $\mathbf{d}$, CALB hydrolytic activity curves of deletion strains. The control strains were GS115/CALB-GCW61 and ku70 $\Delta / C A L B-G C W 61$

immune system, presenting antitumor, antiinflammatory, antimicrobial, wound healing, weight loss, and antidiabetic properties $[25,26]$. $\beta-1,3$-Glucan is also a valuable microbiological binder of mycotoxins that decreases their toxic effects and mediates their removal from media [27, 28]. Mannoproteins were reported to contribute to wine quality, including provide protection against protein and tartaric instability, reduction of astringency, retention of aromatic compounds, and increased body and mouth feel, which are especially appreciated in red wines [29]. Therefore, interest in industrial production of cell wall polysaccharides has been increasing. At present, the usual methods used to increase the content of yeast polysaccharides include the optimization of fermentation conditions, regulation of cell osmotic pressure or mutagenesis breeding [30-32].

In this deletion library, the remodeling of cell wall polysaccharides resulting from certain GPI protein knockouts led to some promising strains, such as the strain ku70 $\Delta$ gcw8 $\Delta$ with a higher glucan content and strain gcw1 $\Delta$ with a higher mannan content. It is also surprising that the glucan and mannan contents of two strains, ku70 $\Delta \mathrm{gcw} 51 \Delta$ and $\mathrm{ku} 70 \Delta \operatorname{gcw} 54 \Delta$, were increased. The gene GCW8 was predicted to be a mannosidase that was capable of catalyzing the hydrolysis of mannose nonreducing residues. In this study, the gene GCW8 was redundant because there were no obvious abnormalities in cell morphology or growth with the strain gcw8 $\Delta$, but the gene was involved in the regulation of cell wall structure synthesis. The gene GCW8 has higher homology with the DCW1 and DFG5 proteins of $S$. cerevisiae, both of these proteins play important roles in cell wall protein cross-linking to the cell wall reticular structure [33]. In S. cerevisiae, there were no abnormal changes in cell growth or cell morphology when these two genes were knocked out individually $[34,35]$. The strain dcw $1 \Delta$ was hypersensitive to zymolyase, which is a cell wall-digesting enzyme, leading to 
defects in cell wall structure. This result indicated that the gene dcw1 is involved in the regulation of cell wall structure. Further studies suggested that both Dcw1p and Dfg5p are GPI-anchored membrane proteins that are required for normal biosynthesis of the cell wall [36]. Abhiram knocked out the genes DCW1 and DFG5 in Neurospora crassa separately, the cell wall proteins would be slight released into the medium. When the two genes were knocked out simultaneously, the release of cell wall proteins into the medium greatly increased, the content of mannan in the cell wall decreased from 14.8 to $2.3 \%$, and the content of glucan increased from 72.8 to $92.6 \%$ [33]. The gene GCW1, also known as GAS1 in P. pastoris, is a $\beta$-1,3-glucanosyltransferase that catalyzes a transglycosylation with $\beta-1,3$-glucan as a substrate. In S. cerevisiae, the loss of Gas1p resulted in the release of $\beta$-1,3-glucan into the medium and an increase in chitin and mannan content in the cell wall. This result was consistent with our finding that the content of mannan was increased by $34 \%$ in strain gcw $1 \Delta$. It is speculated that the higher mannan content is due to a higher expression of cell wall mannoproteins, which might be part of a general response of the cell to cell wall defects in an attempt to prevent cell lysis [37]. In P. pastoris, the morphology of gas1 $\Delta$ was identical to that of $S$. cerevisiae [38]. The genes GCW51 and GCW54 have been little studied, and their physiologic functions were unknown, but according to the above data, both genes must have at least one function, e.g., playing a structural role, contributing to the biosynthesis or assembly of the major cell wall components, or playing a part in cell wall remodeling. Their detailed features will be further explored in the future.

The display of lipase on the cell surface as a whole-cell catalyst is currently a very active topic in organic bioconversion. The hydrophobic environment is commonly believed to be well suited for lipase catalysis because of the "interfacial activation" mechanism [39, 40], but the surfaces of yeast cells are commonly relatively hydrophilic due to the presence of various polysaccharides and proteins. This possibly causes contact resistance between enzyme molecules and hydrophobic substrates [41], and hydrophilic byproducts such as glycerol and water are absorbed on the cell surface and form a hydrophilic layer, leading to cell aggregation and poor dispersibility [42]. To alleviate these problems, hydrophobins are displayed on the $P$. pastoris cell surface; hydrophobins modify the relative hydrophilic properties and improve the catalytic activities of lipase to some extent [43]. Other methods, including coating with ionic liquids and adding decane as a hydrophobic carbon source during fermentation, show higher surface hydrophobicity. Herein, the strains gcw $13 \Delta$, gcw $22 \Delta$, ku70 $\Delta$ gcw30 $\Delta$, and ku70 $\Delta$ gcw53 $\Delta$ attracted attention for their higher surface hydrophobicity. Bioinformatics analysis revealed that the amino acid sequences of the deleted proteins GCW13, GCW22, GCW30, and GCW53 were all highly hydrophilic. Hsu et al. observed clinical isolates of S. cerevisiae with relatively hydrophobic cell surfaces and found that the change in cell wall protein composition was related to the hydrophobicity. The abundances of Scw10p, Pst1p, and Hsp150p/Pir2p, which are all cell wall proteins in the clinical isolates, were at least twofold higher than those in the S288c lab strain [44]. It is speculated that the absence of these proteins leads to the remodeling of cell wall structures, including the cell wall protein quantity and type, and finally the distribution of cell surface hydrophobicity.

To investigate the application potential of the strains with higher surface hydrophobicity, the lipase CALB was displayed with the same anchor, GCW61, on these above strains. Interestingly, the hydrolytic activities of strains gcw22 $\Delta /$ CALB-GCW61, ku70 $\Delta$ gcw30 $\Delta /$ CALB-GCW61 and ku70 $\Delta$ gcw53 $\Delta /$ CALB-GCW61 increased by 69,34 and $20 \%$, respectively, but there was no obvious change in the lipase hydrolytic activity in strain gcw13 $\Delta / C A L B-$ GCW61. We inferred that lipases immobilized on the cell surfaces of these hydrophobic yeast were similar to those immobilized on other hydrophobic carriers; the more hydrophobic environment kept the conformations of the displayed CALB open, and finally, the activities of CALB were affected. It is also desirable to exploit strains with high surface hydrophobicity in other fields, such as wastewater treatment [45] and bioremediation of contaminated sites [46].

\section{Conclusions}

A total of 45 P. pastoris strains with deletions in genes encoding predicted GPI proteins were constructed and showed different characteristics. Among them, some deletions appeared to increase tolerance to methanol, some deletions showed an increase in wall polysaccharides, and others showed surface hydrophobicity. These promising deletion strains not only showed potential applications as production strains but also offered more possibilities to study more functions of GPI proteins, especially putative ones.

\section{Methods}

Strains, plasmids and culture conditions

$P$. pastoris GS115, which was used as a parental strain for gene disruption, and the plasmid pPICZ $\alpha \mathrm{A}$, which was used as the vector, were both purchased from Invitrogen (Carlsbad, CA, USA). The yeast cells were grown in media with different carbon sources for growth analysis, including complex medium $(1 \%(\mathrm{w} / \mathrm{v})$ yeast extract, $2 \%(\mathrm{w} / \mathrm{v})$ peptone) supplemented with $2 \%(\mathrm{w} / \mathrm{v})$ glucose (YPD), 2\% (w/v) glycerol (YPG), and 1, 2\%, or $3 \%(\mathrm{w} / \mathrm{v})$ 
methanol (YPM). The buffered glycerol complex (BMGY) contained $1 \%(\mathrm{w} / \mathrm{v})$ yeast extract, $2 \%(\mathrm{w} / \mathrm{v})$ peptone, $1.34 \%(\mathrm{w} / \mathrm{v})$ yeast nitrogen base, $1 \%(\mathrm{v} / \mathrm{v})$ glycerol and $50 \mathrm{mM}$ potassium phosphate buffer. The buffered methanol complex (BMMY) was the same as BMGY but $1 \%$ glycerol was substituted with methanol. The BMGY and BMMY culture media were used for growth and induction studies, respectively. All yeast strains were cultured at $30^{\circ} \mathrm{C}$.

\section{Construction of the GPI protein deletion library}

Plasmid pPICZ $\alpha \mathrm{A}$ was used as a vector to construct plasmid pPICZC according to the method described by Pan et al. [47]; pPICZC carries the expression cassette of Cre recombinase and the selective marker gene for zeocin resistance. Using the plasmid pPICZC as template and P3 with the lox71 site and P4 with the lox66 site as primers, the modular lox71-Cre-ZeoR-lox66 (CORE) cassette was amplified by PCR. To delete a target gene, the upstream (by primers $\mathrm{P} 5 / \mathrm{P} 6$ ) and downstream (by primers $\mathrm{P} 7 / \mathrm{P} 8$ ) homologous arms of the target gene were individually amplified from $P$. pastoris GS115 genomic DNA by PCR. The reverse primer amplifying the Up-arm fragment and the forward primer amplifying the Down-arm fragment shared 30-40 nucleotides with the CORE cassette. The gene disruption cassettes (Up homologous-lox71-Cre-ZeoR-lox66-Down homologous) were constructed by fusing the CORE cassette with two homologous fragments flanking the region. The disruption cassettes were introduced into the parental strain GS115. After the clones were screened on YPDSZ (YPD plus $1 \mathrm{M}$ sorbitol and $50 \mathrm{mg} / \mathrm{L}$ zeocin) plates and verified by genomic PCR analysis, the Cre-mediated recombination between lox71 and lox66 was subsequently removed through a methanol induction step in YPM medium using two outer primers, P9/P10. To improve the homologous recombination efficiency, the endogenous gene KU70 homologue in GS115 as a host was deleted using this Cre/loxP system. Thereafter, the potential GPIanchored proteins selected from the ORFs of the $P$. pastoris GS115 genome were knocked out according to the method of Zhang et al. [11]; thus, a single GPI gene deletion library was obtained. The sequences of all the primers are provided in Supplementary Table S1.

\section{Growth analysis on different carbon sources}

The deletion strains in GPI-anchored proteins were cultured in 96-well master plates at $30^{\circ} \mathrm{C}$ to obtain a growth curve. The cultures included YPG medium with glycerol as the carbon source, YPD medium with glucose as the carbon source and YPM medium with methanol as the carbon source. To characterize cell growth, the specific growth rate, $\mu$, was estimated using graphical methods from a linear regression of the natural logarithm of the number of viable cells versus time. It was calculated from the following equation: $\mu=\left(\right.$ In $N_{2}$ $\left.\mathrm{InN}_{1}\right) /\left(\mathrm{t}_{2}-\mathrm{t}_{1}\right)$. The growth of strains in BMMY medium with methanol added every $24 \mathrm{~h}$ was recorded.

\section{Measurement of polysaccharide content in the cell wall}

The cell wall polysaccharide was measured as described by François J M with some modifications [48]. To extract cell wall polysaccharides, cells were collected and resuspended in $1 \mathrm{~mL}$ of cold water. After centrifugation at $10,000 \times \mathrm{g}$ for $5 \mathrm{~min}$, the cell pellet was resuspended in $750 \mu \mathrm{L}$ of buffer $\mathrm{TE}$, and $0.73 \mathrm{~g}$ of acid-washed glass beads were added. Disruption of the cells was performed with a mechanical bead beater set at full speed for eight $30 \mathrm{~s}$ periods alternating with $30 \mathrm{~s}$ intervals on ice. The glass beads were washed at least five times with $1 \mathrm{~mL}$ of TE buffer by vortexing the cell suspension briefly and then spinning down the beads ( $500 \mathrm{~g}$ for $1 \mathrm{~min}$ ) after each wash. When the washing solution became limpid, most of the cell debris was no longer bound to the beads. All the washing solutions were centrifuged at $4800 \times \mathrm{g}$ for $15 \mathrm{~min}$, and the cell wall fragments were then freeze-dried. Finally, $10 \mathrm{mg}$ cell wall fragments were weighed accurately, wetted with $75 \mu \mathrm{L}$ of $72 \% \mathrm{H}_{2} \mathrm{SO}_{4}$ solution for $3 \mathrm{~h}$ at room temperature and diluted to $2 \mathrm{~N}$ $\mathrm{H}_{2} \mathrm{SO}_{4}$ for $4 \mathrm{~h}$ at $100^{\circ} \mathrm{C}$. The acid solution was neutralized with saturated $\mathrm{Ba}(\mathrm{OH})_{2}$ until the $\mathrm{pH}$ was neutral, and the final volume was adjusted to $20 \mathrm{~mL}$ with Milli-Q water. The solution was centrifuged at $4800 \times \mathrm{g}$ for 15 $\min$ at $4{ }^{\circ} \mathrm{C}$. One milliliter of the supernatant was taken for HPIC measurement according to François J M [48].

\section{Measurement of cell surface hydrophobicity}

The hydrophobic nature of the outermost surface of the yeast cells was determined by microbial adhesion to hydrocarbon (MATH), as described by Hama et al. [41] and modified by Wang et al. [43]. In this method, the proportion of yeast cells passing into the n-butyl alcohol reflects the adsorption of the yeast cells to the organism. The cells were washed twice and resuspended in PBS to an $\mathrm{OD}_{600}$ of 2 , and the accurate value was defined as $\mathrm{A}_{1}$. A $2 \mathrm{~mL}$ aliquot of butyl alcohol was then added to $2 \mathrm{ml}$ of the cell suspension, vortexed for $30 \mathrm{~s}$ and allowed to stand for $3 \mathrm{~min}$ to enable the complete separation of the two phases. The lower aqueous phase was measured at $600 \mathrm{~nm}$ and defined as $A_{2} . A_{1}$ and $A_{2}$ were measured using a spectrophotometer (BIO-RAD, USA). Hydrophobicity was then given as a percentage calculated from the following equation: hydrophobicity $(\%)=\left(A_{1}-A_{2}\right) / A_{1}$. The average hydrophobicity expressed as the grand average of hydropathy (GRAVY) value [49] for identified proteins and peptides was calculated using Prot-Param software available at https://web.expasy.org/protparam/. 


\section{Analysis of CALB hydrolytic activity}

The plasmid pZCALB-GCW61 containing mature CALB cDNA [50] was transformed into strains GS115 and GS115 ku70 $\Delta$ as the control strains. CALB hydrolytic activity was assayed using a modified method [11]. The substrate $P$-nitrophenyl butyrate (pNPB; Sigma, St. Louis, MO, USA), which was the substrate, was emulsified by sonication in ultrapure water containing $0.5 \%$ Triton X-100, resulting in a final concentration of 25 $\mathrm{mM}$. The $1 \mathrm{~mL}$ reaction system contained $940 \mu \mathrm{L}$ of Tris- $\mathrm{HCl}$ buffer $(50 \mathrm{mM}, \mathrm{pH} 8.0), 50 \mu \mathrm{L}$ of pNPB, and $10 \mu \mathrm{L}$ of cell supernatant with the appropriate dilution to ensure the absorbance was in a reasonable range. Then, the system was incubated for $5 \mathrm{~min}$ at $45^{\circ} \mathrm{C}$. Finally, the assay mixture was centrifuged at $6000 \times \mathrm{g}$ for $1 \mathrm{~min}$. Using a kinetic microplate reader (Molecular Devices, Sunnyvale, CA, USA), the absorbance of $200 \mu \mathrm{L}$ supernatant was measured at $405 \mathrm{~nm}$ with a 96-well plate. The hydrolytic activity of CALB was defined as the amount of enzyme required to release $1 \mu \mathrm{mol}$ pNP per min under the assay conditions. Average values were generated from triplicates of each sample.

\section{Supplementary information}

Supplementary information accompanies this paper at https://doi.org/10. 1186/s12866-020-01928-y.

Additional file 1: Table S1. Primers used in this study.

\section{Abbreviations}

GPI: Glycosylphosphatidylinositol; CALB: Candida antarctica lipase B; CWP: Cell wall protein; CWI: Cell wall integrity; NHEJ: Non-homologous-endjoining; MATH: Microbial adhesion to hydrocarbon; pNPB: p-nitrophenyl butyrate; pNP: p-nitrophenol

\section{Acknowledgements}

We appreciate the assistance of the staff at the Guangdong Key Laboratory of Fermentation and Enzyme Engineering at South China University of Technology.

\section{Authors' contributions}

Conceived and designed the experiments: LY and HSY. Performed the experiments: WP, ZCJ. Supervision: LSL and ZSP. The manuscript was written by WP. Review and editing: HSY and ZFG. HSY acquired the funds that were used in the study. All authors read and approved the final manuscript.

\section{Funding}

This work was supported by the grants from the National Natural Science Foundation of China (No. 31771958). The funding body had no role in study design, data collection, analysis and interpretation, and in writing of the manuscript.

\section{Availability of data and materials}

The datasets used and analysed during the current study are available from the corresponding author on reasonable request.

\section{Ethics approval and consent to participate}

Not applicable.

\section{Consent for publication}

Not applicable.

\section{Competing interests}

We declare that we have no competing interests.

Received: 9 March 2020 Accepted: 29 July 2020

Published online: 24 August 2020

\section{References}

1. Caras IW, Weddell GN, Davitz MA, Nussenzweig V, Martin DW. Signal for attachment of a phospholipid membrane anchor in decay accelerating factor. Science. 1987;238(4831):1280-3.

2. Leidich SD, Drapp DA, Orlean P. A conditionally lethal yeast mutant blocked at the first step in glycosyl phosphatidylinositol anchor synthesis. J Biol Chem. 1994;269(14):10193-6.

3. Pittet M, Conzelmann A. Biosynthesis and function of GPI proteins in the yeast Saccharomyces cerevisiae. Biochim Biophys Acta. 2007;1771(3):405-20.

4. Levin DE. Regulation of cell wall biogenesis in Saccharomyces cerevisiae: the cell wall integrity signaling pathway. Genetics. 2011;189(4):1145-75.

5. Popolo L, Gualtieri T, Ragni E. The yeast cell-wall salvage pathway. Med Mycol. 2001;39(1):111-21.

6. Klis FM, Boorsma A, De Groot PWJ. Cell wall construction in Saccharomyces cerevisiae. Yeast (Chichester, England). 2006;23(3):185-202.

7. Pardo M, Monteoliva L, Vázquez P, Martínez R, Molero G, Nombela C, et al, PST1 and ECM33 encode two yeast cell surface GPI proteins important for cell wall integrity. Microbiology. 2004;150(12):4157-70.

8. Zhang J, Astorga MA, Gardner JM, Walker ME, Grbin PR, Jiranek V. Disruption of the cell wall integrity gene ECM33 results in improved fermentation by wine yeast. Metab Eng. 2018;45:255-64.

9. Cho EY, Cheon SA, Kim H, Choo J, Lee D-J, Ryu HM, et al. Multiple-yapsindeficient mutant strains for high-level production of intact recombinant proteins in Saccharomyces cerevisiae. J Biotechnol. 2010;149(1-2):1-7.

10. Bamba T, Inokuma K, Hasunuma T, Kondo A. Enhanced cell-surface display of a heterologous protein using SED1 anchoring system in SED1-disrupted Saccharomyces cerevisiae strain. J Biosci Bioeng. 2018;125(3):306-10.

11. Zhang L, Liang S, Zhou X, Jin Z, Jiang F, Han S, et al. Screening for glycosylphosphatidylinositol-modified cell wall proteins in Pichia pastoris and their recombinant expression on the cell surface. Appl Environ Microb. 2013;79(18):5519-26.

12. Yang Z, Zhang Z. Engineering strategies for enhanced production of protein and bio-products in Pichia pastoris: a review. Biotechnol Adv. 2018; 36(1):182-95.

13. Juturu V, Wu JC. Heterologous protein expression in Pichia pastoris: latest research Progress and applications. Chembiochem. 2018;19(1):7-21.

14. Inokuma $\mathrm{K}$, Kurono $\mathrm{H}$, den Haan $\mathrm{R}$, van Zyl WH, Hasunuma $\mathrm{T}$, Kondo A. Novel strategy for anchorage position control of GPI-attached proteins in the yeast cell wall using different GPI-anchoring domains. Metab Eng. 2020; 57:110-7.

15. Gupta SK, Shukla P. Advanced technologies for improved expression of recombinant proteins in bacteria: perspectives and applications. Crit Rev Biotechnol. 2016;36(6):1089-98.

16. Dangi AK, Dubey KK, Shukla P. Strategies to improve Saccharomyces cerevisiae: technological advancements and evolutionary engineering. Indian J Microbiol. 2017;57(4):378-86.

17. Näätsaari L, Mistlberger B, Ruth C, Hajek T, Hartner FS, Glieder A. Deletion of the Pichia pastoris KU70 homologue facilitates platform strain generation for gene expression and synthetic biology. PLoS One. 2012;7(6):e39720-32.

18. Mattanovich D, Branduardi P, Dato L, Gasser B, Sauer M, Porro D. Recombinant protein production in yeasts. Methods Mol Biotechnol. 2012; 824:329-58

19. Stratton J, Chiruvolu V, Meagher M. High cell-density fermentation. Methods Mol Biol. 1998;103:107-20.

20. Sinha J, Plantz BA, Zhang W, Gouthro M, Schlegel V, Liu CP, et al. Improved production of recombinant ovine interferon- $\tau$ by Mut ${ }^{+}$strain of Pichia pastoris using an optimized methanol feed profile. Biotechnol Prog. 2003; 19(3):794-802

21. Yasokawa D, Murata S, Iwahashi Y, Kitagawa E, Nakagawa R, Hashido T, et al. Toxicity of methanol and formaldehyde towards Saccharomyces cerevisiae as assessed by DNA microarray analysis. Appl Biochem Biotechnol. 2010; 160(6):1685-98.

22. Zou C, Pan W, Liang S, Han S, Zheng S, Lin Y. Deletion of the GCW13 gene derepresses Gap1-dependent uptake of amino acids in Pichia pastoris 
grown on methanol as the sole carbon source. Biochem Biophys Res Commun. 2018;501:226-31.

23. Leßmeier L, Wendisch VF. Identification of two mutations increasing the methanol tolerance of Corynebacterium glutamicum. BMC Microbiol. 2015 15(1):216-37.

24. Auesukaree C, Damnernsawad A, Kruatrachue M, Pokethitiyook P, Boonchird C, Kaneko Y, et al. Genome-wide identification of genes involved in tolerance to various environmental stresses in Saccharomyces cerevisiae. J Appl Genet. 2009;50(3):301-10.

25. Varelas V, Liouni M, Calokerinos AC, Nerantzis ET. An evaluation study of different methods for the production of $\beta$-D-glucan from yeast biomass. Drug Test Anal. 2016;8(1):46-55.

26. Stier $\mathrm{H}$, Ebbeskotte $\mathrm{V}$, Gruenwald J. Immune-modulatory effects of dietary yeast Beta-1,3/1,6-D-glucan. Nutr J. 2014;13(1):38-46.

27. Caridi A. New perspectives in safety and quality enhancement of wine through selection of yeasts based on the parietal adsorption activity. Int J Food Microbiol. 2007:120(1-2):167-72.

28. Kogan $\mathrm{G}$, Kocher $\mathrm{A}$. Role of yeast cell wall polysaccharides in pig nutrition and health protection. Livest Sci. 2007;109(1):161-5.

29. Giovani G, Canuti V, Rosi I. Effect of yeast strain and fermentation conditions on the release of cell wall polysaccharides. Int J Food Microbiol. 2010;137(23):303-7.

30. Liu H-Z, Wang Q, Liu Y-Y, Fang F. Statistical optimization of culture media and conditions for production of mannan by Saccharomyces cerevisiae. Biotechnol Bioprocess Eng. 2009;14(5):577-83.

31. Liu HZ, Wang Q, Liu XY, Tan S-S. Effects of spaceflight on polysaccharides of Saccharomyces cerevisiae cell wall. Appl Microbiol Biotechnol. 2008;81(3): 543-50.

32. Wang Y, Yao S, Wu T. Combination of induced autolysis and sodium hypochlorite oxidation for the production of Saccharomyces cerevisiae (13)-B-D-glucan. World J Microbiol Biotechnol. 2003;19(9):947-52.

33. Maddi A, Fu C, Free SJ. The Neurospora crassa dfg 5 and dew1 genes encode $a-1,6-$ mannanases that function in the incorporation of glycoproteins into the cell wall. PLoS One. 2012;7(6):e38872-80.

34. Mösch HU, Fink GR. Dissection of filamentous growth by transposon mutagenesis in Saccharomyces cerevisiae. Genetics. 1997;145(3):671-84.

35. Winzeler EA, Shoemaker D, Astromoff A, Liang H, Anderson K, André B, et al. Functional characterization of the $\mathrm{S}$. cerevisiae genome by gene deletion and parallel analysis. Science. 1999;285:901-6.

36. Kitagaki H, Wu H, Shimoi H, Ito K. Two homologous genes, DCW1 (YKL046c) and DFG5, are essential for cell growth and encode glycosylphosphatidylinositol (GPI)-anchored membrane proteins required for cell wall biogenesis in Saccharomyces cerevisiae. Mol Microbiol. 2002; 46(4):1011-22

37. Ram AF, Kapteyn JC, Montijn RC, Caro LH, Douwes JE, Baginsky W, et al. Loss of the plasma membrane-bound protein Gas1p in Saccharomyces cerevisiae results in the release of beta1,3-glucan into the medium and induces a compensation mechanism to ensure cell wall integrity. J Bacteriol. 1998;180(6):1418-24.

38. Marx H, Sauer M, Resina D, Vai M, Porro D, Valero F, et al. Cloning, disruption and protein secretory phenotype of the GAS1 homologue of Pichia pastoris. FEMS Microbiol Lett. 2006;264(1):40-7.

39. Zaks A, Klibanov AM. Enzyme-catalyzed processes in organic solvents. P Natl Acad Sci USA. 1985:82(10):3192-6.

40. Palomo JM, Peñas MM, Fernández-Lorente G, Mateo C, Pisabarro AG, Fernández-Lafuente $\mathrm{R}$, et al. Solid-phase handling of hydrophobins: immobilized hydrophobins as a new tool to study lipases. Biomacromolecules. 2003:4(2):204-10.

41. Hama S, Yoshida A, Nakashima K, Noda H, Fukuda H, Kondo A. Surfactantmodified yeast whole-cell biocatalyst displaying lipase on cell surface for enzymatic production of structured lipids in organic media. Appl Microbiol Biotechnol. 2010;87(2):537-43.

42. Jin Z, Han SY, Zhang L, Zheng SP, Wang Y, Lin Y. Combined utilization of lipase-displaying Pichia pastoris whole-cell biocatalysts to improve biodiesel production in co-solvent media. Bioresour Technol. 2013;130:102-9.

43. Wang P, He J, Sun Y, Reynolds M, Zhang L, Han S, et al. Display of fungal hydrophobin on the Pichia pastoris cell surface and its influence on Candida antarctica lipase B. Appl Microbiol Biotechnol. 2016;100(13):5883-95.

44. Hsu PH, Chiang PC, Liu CH, Chang YW. Characterization of Cell Wall proteins in Saccharomyces cerevisiae clinical isolates elucidates Hsp150p in virulence. PLoS One. 2015;10(8):e0135174-91.
45. Guo F, Zhang SH, Yu X, Wei B. Variations of both bacterial community and extracellular polymers: the inducements of increase of cell hydrophobicity from biofloc to aerobic granule sludge. Bioresour Technol. 2011;102(11): 6421-8.

46. Zhang J, Sun Z, Li Y, Peng X, Li W, Yan Y. Biodegradation of p-nitropheno by Rhodococcus sp. CN6 with high cell surface hydrophobicity. J Hazard Mater. 2009;163(2-3):723-8.

47. Pan R, Zhang J, Shen W-L, Tao Z-Q, Li S-P, Yan X. Sequential deletion of Pichia pastoris genes by a self-excisable cassette. FEMS Yeast Res. 2011;11(3): 292-8.

48. François JM. A simple method for quantitative determination of polysaccharides in fungal cell walls. Nat Protoc. 2006;1(6):2995-3000.

49. Kyte J, Doolittle RF. A simple method for displaying the hydropathic character of a protein. J Mol Biol. 1982;157(1):105-32.

50. Wang $P$, Zhang L, Fisher R, Chen M, Liang S, Han S, et al. Accurate analysis of fusion expression of Pichia pastoris glycosylphosphatidylinositol-modified cell wall proteins. J Ind Microbiol Biotechnol. 2017;44(9):1355-65.

\section{Publisher's Note}

Springer Nature remains neutral with regard to jurisdictional claims in published maps and institutional affiliations.

\section{Ready to submit your research? Choose BMC and benefit from:}

- fast, convenient online submission

- thorough peer review by experienced researchers in your field

- rapid publication on acceptance

- support for research data, including large and complex data types

- gold Open Access which fosters wider collaboration and increased citations

- maximum visibility for your research: over $100 \mathrm{M}$ website views per year

At $\mathrm{BMC}$, research is always in progress.

Learn more biomedcentral.com/submissions 